Article

\title{
Self-Expandable Retainer for Endoscopic Visualization in the External Auditory Canal: Proof of Concept in Human Cadavers
}

\author{
Yehree Kim ${ }^{1,+}$, Jeon Min Kang ${ }^{2,+}$, Ho-Young Song ${ }^{3,4}$, Woo Seok Kang ${ }^{1}$, Jung-Hoon Park ${ }^{2, *, \ddagger}$ \\ and Jong Woo Chung $1, *, \neq($ CD \\ 1 Department of Otorhinolaryngology-Head \& Neck Surgery, Asan Medical Center, University of Ulsan \\ College of Medicine, 88 Olympic-ro 43-gil, Songpa-gu, Seoul 05505, Korea; yehreek@hotmail.com (Y.K.); \\ entkang7@gmail.com (W.S.K.) \\ 2 Biomedical Engineering Research Center, Asan Institute for Life Scineces, Asan Medical Center, \\ 88 Olympic-ro 43-gil, Songpa-gu, Seoul 05505, Korea; miny2208@naver.com \\ 3 Department of Radiology, Asan Medical Center, University of Ulsan College of Medicine, \\ 88 Olympic-ro 43-gil, Songpa-gu, Seoul 05505, Korea; hysong@amc.seoul.kr \\ 4 Department of Radiology, UT Health Science Center at San Antonio, 7703 Floyd Curl Drive, \\ San Antonio, TX 78229, USA \\ * Correspondence: jhparkz@amc.seoul.kr (J.-H.P.); jwchung@amc.seoul.kr (J.W.C.) \\ + These authors contributed equally to this work. \\ $\ddagger \quad$ These authors contributed equally to this work.
}

Received: 12 February 2020; Accepted: 4 March 2020; Published: 10 March 2020

\begin{abstract}
This study was conducted to investigate the efficacy of a self-expandable retainer (SER) for endoscopic visualization of the external auditory canal (EAC). Tympanomeatal flap (TMF) elevation was performed in six cadaveric heads. Two different types of SER were placed. The procedural feasibility was assessed by endoscopic images. Technical success rate, procedure time, endoscopy lens cleaning, and presence of mucosal injuries were analyzed. TMF elevation and SER placement were successful in all specimens and there were no procedure-related complications. The mean procedure time with the SERs was significantly shorter than without $(p<0.001)$. The mean number of times the endoscopy lens was cleaned during the procedure was significantly lower in the SER group $(p<0.001)$. In the SER group, endoscopy insertion into the EAC was easier without tissue contact with the lens during the TMF elevation compared with the non-SER group. There were no mucosal injuries. SER placement is effective for endoscopic visualization via the expanded and straightened EAC. A fully covered type of SER is preferable. The device can be useful for endoscopic ear surgery, reducing procedure time and reducing the need for endoscopy lens cleaning during the procedure.
\end{abstract}

Keywords: self-expandable metallic stent; ear canal; cadaver; ear speculum; endoscopy

\section{Introduction}

The past three decades have witnessed a growth in the use of endoscopy and it is used in several surgical disciplines including otologic surgery [1]. Endoscopic ear surgery (EES) has several advantages over conventional surgical microscopy. There is a wider field of view, improved resolution with high magnification, and the ability to "see around corners," allowing direct visualization of normally hidden recesses [2]. The external auditory canal (EAC) is typically used as the approach for surgery, obviating the need to create access pathways or retract soft tissues [3-5].

The EAC is about $2.5 \mathrm{~cm}$ in length and comprises a lateral cartilaginous portion and a medial bony portion $[6,7]$. There is a natural curvature to the external meatus which plays little role in endoscopy 
since the endoscope is positioned medial to the curvature [8]. However, the cartilaginous and bony portions need to be aligned to insert the endoscope. The cartilaginous portion of the EAC is often pushed posteriorly using the shaft of the endoscope. Certain techniques such as the Tarabichi's stitch can be used to straighten the EAC and increase the working space for the endoscope $[9,10]$.

Because the space in the EAC and middle ear is limited, a clean surgical field is required to assure patient safety and benefit from the wide-angle vision offered by the endoscope [11]. The lens of the endoscope is easily smeared by the hair and earwax in the EAC and blood, however minimal, can obscure the view. The authors, therefore, devised a self-expandable retainer (SER) which can be placed in the EAC to aid surgery.

An ideal retainer would provide straight and clean entry to the middle ear without causing damage to the soft tissues of the EAC and, further, protect the tissues from trauma by the surgical instruments. The aim of this study was to investigate the efficacy and technical feasibility of SERs for endoscopic visualization in the EAC of cadaveric heads. SER placement was found to be effective for endoscopic visualization due to the expansion and straightening of the EAC.

\section{Materials and Methods}

\subsection{Study Design}

Twelve EACs of six adult human cadaveric heads were used in this study. None of them had a history of ear surgery, ear disease, or trauma. A tympanomeatal flap (TMF) was elevated in both ears of all the cadaveric specimens. One of the two available types of SER was placed in the left ear before the TMF elevation was performed. The bare type was placed in three cadaveric heads and the fully covered type of SER was placed in the remaining three. The right-sided ear had just the TMF elevation procedure without the placement of an SER.

\subsection{Self-Expandable Retainers}

Two different types of SERs were used (S\&G Biotech, Yongin, Korea) (Figure 1): bare and fully covered types.

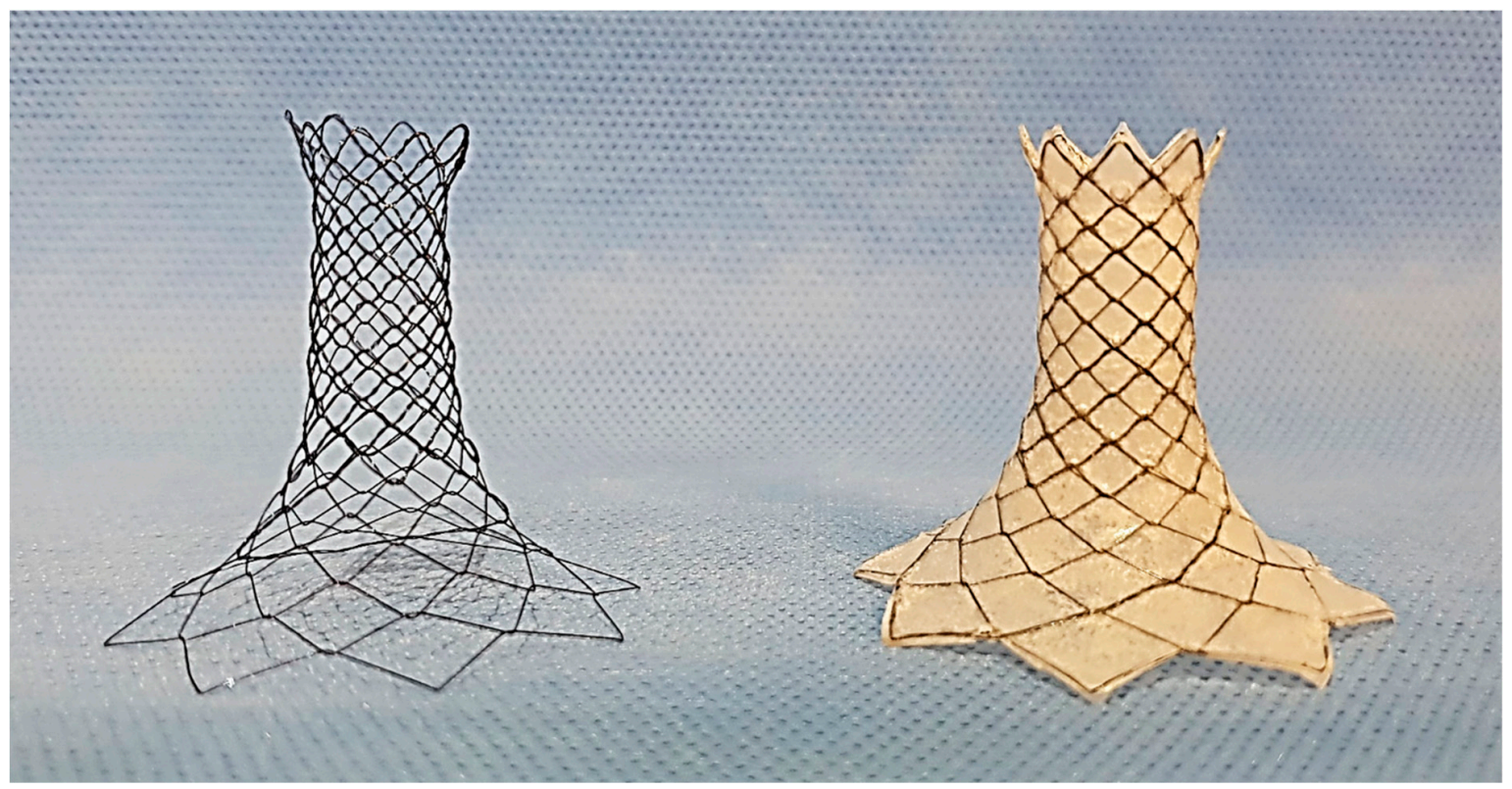

Figure 1. Photograph showing self-expandable retainers (SERs). Left: bare type of SER. Right: fully covered type of SER. 
The SER comprises two parts: a straight self-expandable metallic stent (SEMS) part for the EAC and a flared part for the pinna. The straight SEMS part is knitted from a $0.127-\mathrm{mm}$ nitinol wire into a tubular configuration. When fully expanded, the straight SEMS is $6 \mathrm{~mm}$ in diameter and $12 \mathrm{~mm}$ in length. The flared end, designed to be in direct contact with the pinna for endoscope insertion, is a round flared mesh made from a $0.127-\mathrm{mm}$ nitinol wire. The end of the flared section is $40 \mathrm{~mm}$ in diameter and $23 \mathrm{~mm}$ in length and is spirally connected to the straight SEMS part. The total length of the SER is $35 \mathrm{~mm}$. A fully covered variant of the SER is coated with silicone (MED-6640, Carpinteria, CA, USA) using the dipping method. The SERs were placed using forceps.

\subsection{Tympanomeatal Flap Elevation and SER Placement}

The cadaver head was turned to the side position. Using forceps, a collapsed SER was advanced through the pinna of the left EAC under endoscopic guidance (VISERA 4K UHD Rhinolaryngoscope; Olympus, Tokyo, Japan). The proximal end of the SER was located just lateral to the tympanic membrane. The TMF elevation using a canal knife was performed under endoscopic guidance with SER in place (left ear) or without SER (right ear). The SERs were immediately removed using forceps after the TMF elevation. Each specimen was carefully evaluated for any procedure-related complications using post-procedural endoscopic examination.

\subsection{Study Definition and Data Analysis}

The efficacy and feasibility of the use of SERs were assessed by the technical success rate, procedure time, number of times the endoscopy lens was cleaned, and presence of any mucosal injuries after SER removal, in the cadaveric specimens. Technical success was defined as successful TMF elevation under endoscopic guidance. The procedure time was measured from the insertion of the endoscope into the EAC (with or without the SER) to its removal after completion of the TMF elevation. The procedural feasibility and proximal end location of the SER were analyzed using endoscopic images.

\subsection{Statistical Analysis}

Data are expressed as the mean \pm standard deviation (SD). The differences between the two groups were analyzed using the paired $t$ test. The mean differences and $95 \%$ confidence intervals $(\mathrm{CIs})$ were constructed, as appropriate. A $p$ value of $<0.05$ was considered statistically significant. Statistical analyses were performed using SPSS software, version 23.0 (SPSS, IBM, Chicago, IL, USA).

\section{Results}

TMF elevation was technically successful in all specimens of the adult human cadaveric heads and there were no procedure-related complications. The placement of both types of SER in the left cadaveric ears was technically successful. The proximal ends of the SERs were located 2-3 $\mathrm{mm}$ from the tympanic membrane. The mean $( \pm \mathrm{SD})$ procedure time for TMF elevation with SERs was significantly shorter than that for TMF elevation without SERs (175.6 $\pm 10.3 \mathrm{~s}$ vs. $312.2 \pm 23.4 \mathrm{~s} ; p<0.001)$ (mean difference, $136.5 \mathrm{~s} ; 95 \% \mathrm{CI}, 111.7-161.3 \mathrm{~s})$. The mean $( \pm \mathrm{SD})$ number of endoscopy lens cleanings during the procedure was significantly less in the SER group compared with the non-SER group $(0.83 \pm 0.75$ vs. $5.16 \pm 1.17 ; p<0.001$ ) (mean difference, $4.33 ; 95 \%$ CI, 3.07-5.59) (Figure 2). 

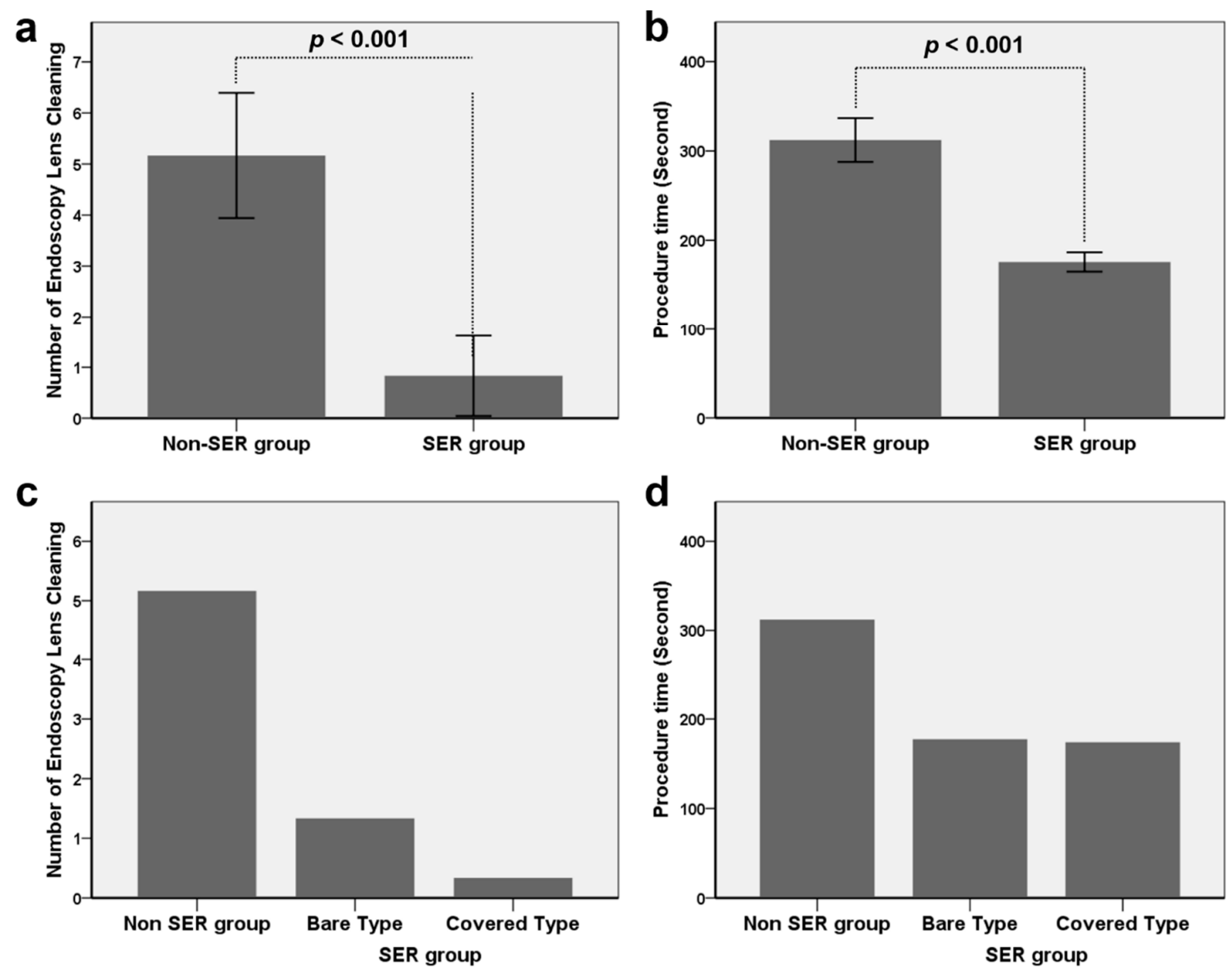

Note. SER: self-expandable retainer

Figure 2. The number of endoscopy lens cleaning interventions $(\mathbf{a}, \mathbf{c})$ and procedure time $(\mathbf{b}, \mathbf{d})$ in the non-SER and SER groups. Data are mean difference with a 95\% confidence interval.

In the SER group, insertion of the endoscope into the EAC was relatively easy without any resistance or tissue contact by the lens during the TMF elevation compared with the non-SER group because of the expanded and straightened EAC (Figure 3).

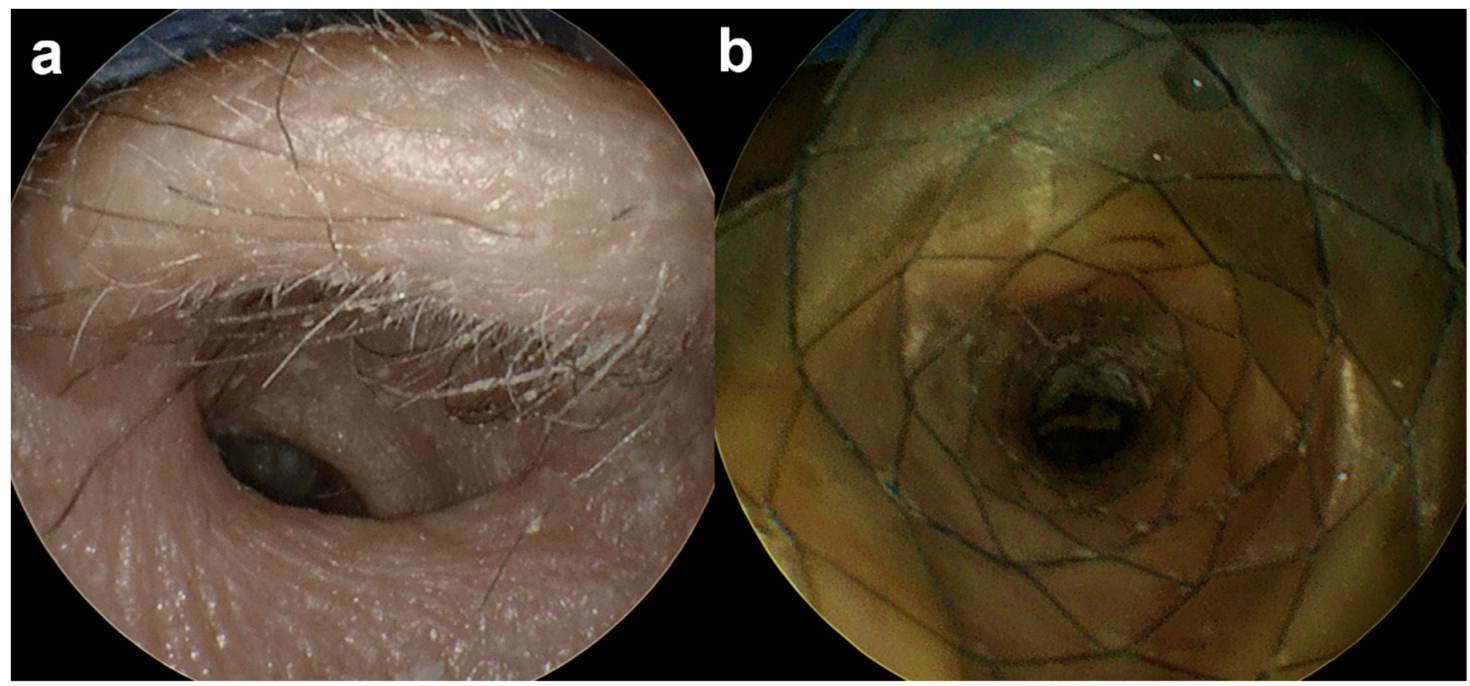

Figure 3. Endoscopic images showing the view at the pinna. (a) Endoscopic image showing hairs and irregular external auditory canal (EAC) anatomy. (b) Endoscopic image taken immediately after fully covered self-expandable retainer placement showing the expanded and straightened EAC. 
However, with the bare type of SER, hairs and foreign bodies in the EAC were visible through the wire mesh of the straight part of the SEMS, (Figure 4).

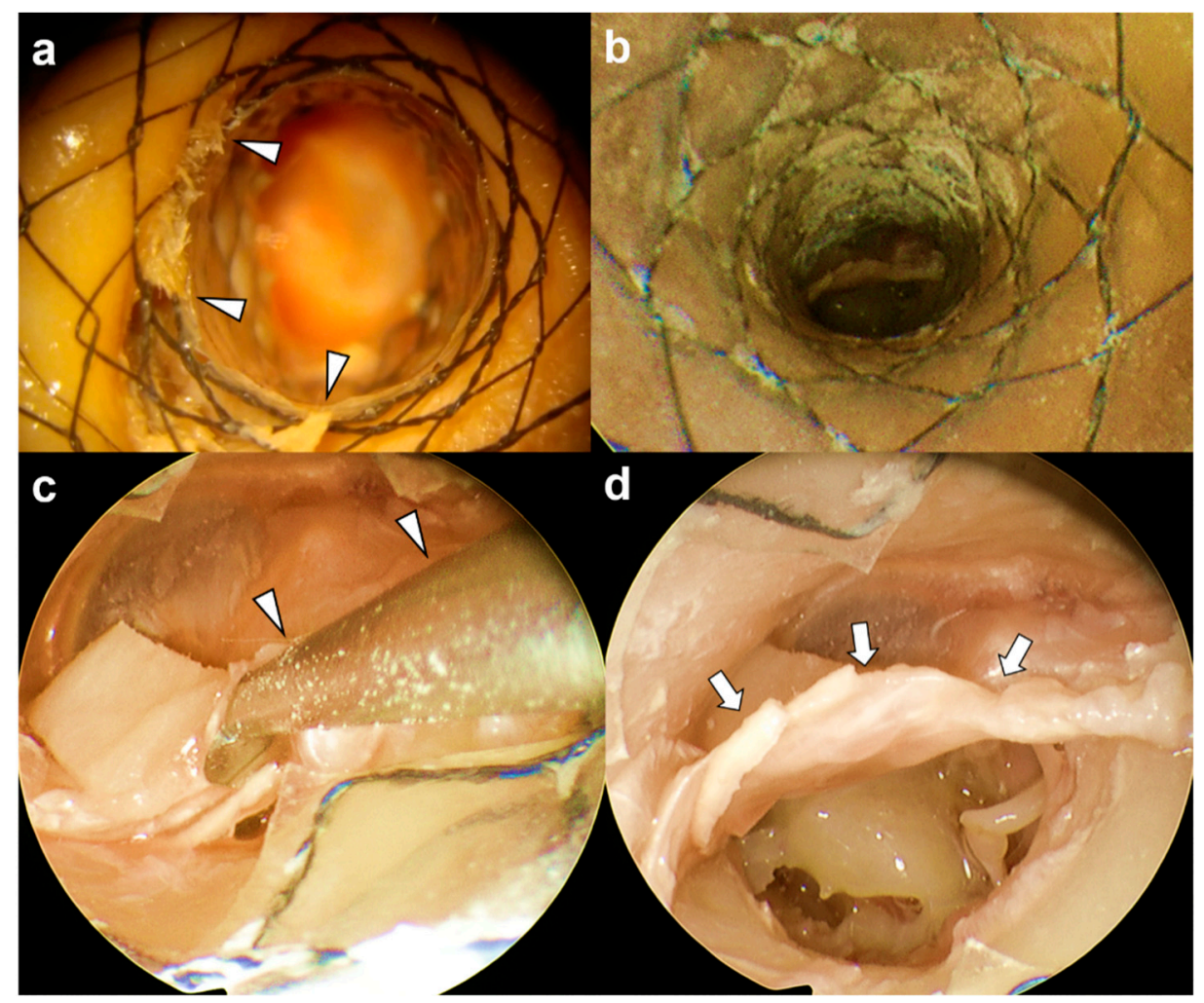

Figure 4. Endoscopic images obtained immediately after tympanomeatal flap (TMF) elevation in the self-expandable retainer (SER) group. (a) Endoscopic image showing the bare type of SER and hairs and earwax (arrowheads) in the external auditory canal visible through the wire mesh of the SER. (b) Endoscopic image showing the fully covered SER. (c) TMF elevation was performed using a canal knife (arrowheads). (d) A successfully elevated tympanic membrane (arrows).

The mean number of times the endoscopy lens was cleaned during the procedure was less in the fully covered SERs group $(0.3 \pm 0.56)$ compared with the bare SERs group $(1.3 \pm 0.57)$ without a statistical difference $(p=0.101)$. There were no mucosal injuries after the procedure in any specimen.

\section{Discussion}

The current study is a cadaveric proof of concept study of SER for better endoscopic visualization in EES. The newly developed SER has several advantages over the conventional ear speculum. First, it is safe and easy to place and remove with the use of forceps. After completing the whole procedure, endoscopic examination revealed no damage to the skin or soft tissue of the EAC.

Second, the use of an SER may allow a significant reduction in the procedure time during various EES due to less frequent endoscopic lens cleansing to remove hair and earwax. As EES is performed by a single surgeon, endoscope contamination can compromise the surgeon's vision and negatively affect the surgery [12]. In a survey asking otologists who routinely perform EES about the need for better instrumentation, more than $60 \%$ of respondents felt the need for improvements in order to 'keep the endoscope lens clean' [13].

The skin which lines the cartilaginous section of the canal is thicker, more mobile, and contains sebaceous and ceruminous glands and hair follicles [14] that can contaminate the lens while inserting the endoscope, requiring the surgeons to intermittently discontinue surgery to remove and clean the endoscope and reinsert it $[15,16]$. This can be time-consuming and taxes the surgeon's patience. Often the canal hair is trimmed before the start of the surgery to reduce fogging and smearing. 
Endoscope contamination can also be due to bleeding. In traditional microscopic surgery, the non-dominant hand holds the suction instrument to maintain retraction and removal of blood from the operative field while the dominant hand holds the instruments [17]. Bleeding in EES is a major concern for the operating surgeon because the non-dominant hand also must hold the endoscope. Techniques utilized to achieve hemostasis include diathermy probes (monopolar and bipolar) [11], diluted epinephrine injection, epinephrine-soaked cotton balls that can also be used as adjuncts for soft tissue dissection [18], and preoperative injection of tranexamic acid [12] which is an anti-fibrinolytic used in a variety of surgical procedures [19-21].

Because our study used cadavers, we could not assess the advantage of the SER with regard to bleeding. The most common site of bleeding is in the posterior and superior portion of the EAC [11], where the main vascular supply comes from the anterior tympanic and deep auricular branches of the internal maxillary artery, the superficial temporal artery, and the posterior auricular artery [22]. We hypothesize that the SER might reduce bleeding by protecting the EAC skin from trauma from sharp surgical instruments and also by pressing against the vascular structures and reducing blood flow by the outward expanding force of the SER putting pressure on the soft tissues of the EAC. This idea needs further verification in preclinical and clinical studies.

Thermal injuries can be caused by the light source of endoscopes [23-28], therefore frequent removal of the endoscope from the field may be beneficial in preventing this [26]. Using an SER will reduce the frequency of endoscope removal from the surgical field; therefore other cooling methods should be implemented to ensure safety. These include the use of suction, turning off the light source at regular intervals, and the use of endoscope sheaths and saline irrigation [26,29].

SER not only allows for a straightened and slightly widened ear canal but also protects the soft tissue around the ear canal from the surgical instruments used in EES. While endoscopes facilitate a broader field of view, bone removal may still be necessary for better visualization and easier access. Osteotomes, curettes, drills, and piezoelectric systems are used for this [13]. When using drills, surgeons have to take care to protect the EAC soft tissue from possible injury by the drill shaft and an SER could be helpful with this.

There are some limitations to this study. First, although the variables of interest reached statistical significance, the sample size was too small to perform a robust statistical analysis. However, the differences in our findings between the SER and non-SER groups were indisputable. Second, we only performed endoscopic inspection to evaluate mucosal injuries after SER removal. Histopathological analysis needs to be used to verify possible mucosal injury. Third, in EES, one side could be easier to work with than the other, according to the surgeon. This matter of dexterity could also have affected the outcome as all SERs were inserted on the left ear in this cadaver study.

To our knowledge, we are the first to devise a tool for protection of the endoscope from contamination and the EAC from surgical trauma to assist in EES. A retainer can be helpful particularly in cases in which a long procedure time or bleeding is expected. Although additional studies are required to investigate the safety and optimal size of the SER, the results of our study support the basic concept of using an SER to enhance the space of the EAC for EES.

\section{Conclusions}

SER placement appears to be effective for endoscopic visualization by expanding and straightening the EAC in a human cadaveric head. A fully covered SER is preferable and the device has great potential for use in EES to reduce procedure time and reduce the frequency of endoscope lens cleaning during surgery.

Author Contributions: J.-H.P. and J.W.C. contributed to this work in study planning, cadaver experiment, data analysis, and manuscript preparation. Y.K. contributed to data analysis and manuscript preparation with critical comments; J.M.K., H.-Y.S., and W.S.K. contributed to the cadaver experiment. All authors have read and agreed to the published version of the manuscript. 
Funding: This research was supported by a grant (2019IE7044) from the Asan Institute for Life Sciences, Asan Medical Center, Seoul, Korea.

Acknowledgments: The authors would like to thank Enago (http://www.enago.co.kr) for the English language review.

Conflicts of Interest: The authors declare no conflict of interest.

\section{References}

1. Kapadiya, M.; Tarabichi, M. An overview of endoscopic ear surgery in 2018. Laryngoscope Investig. Otolaryngol. 2019, 4, 365-373. [CrossRef] [PubMed]

2. Kiringoda, R.; Kozin, E.D.; Lee, D.J. Outcomes in Endoscopic Ear Surgery. Otolaryngol. Clin N. Am. 2016, 49, 1271-1290. [CrossRef] [PubMed]

3. Tarabichi, M. Endoscopic management of acquired cholesteatoma. Am. J. Otol. 1997, 18, 544-549. [PubMed]

4. Tarabichi, M. Endoscopic middle ear surgery. Ann. Otol. Rhinol. Laryngol. 1999, 108, 39-46. [CrossRef]

5. Mitchell, S.; Coulson, C. Endoscopic ear surgery: A hot topic? J. Laryngol. Otol. 2017, 131, 117-122. [CrossRef]

6. Anson, B.J.; Donaldson, J.A. Surgical Anatomy of the Temporal Bone; Saunders: Philadelphia, PA, USA, 1981; Volume 31.

7. Bojrab, D.I.; Bruderly, T.; Abdulrazzak, Y. Otitis externa. Otolaryngol. Clin. N. Am 1996, 29, 761-782.

8. Preyer, S. Endoscopic ear surgery-A complement to microscopic ear surgery. HNO 2017, 65, $29-34$. [CrossRef]

9. Tarabichi, M. Endoscopic transcanal middle ear surgery. Indian J. Otolaryngol. Head Neck Surg. 2010, 62, 6-24. [CrossRef]

10. Parelkar, K.; Thorawade, V.; Marfatia, H.; Shere, D. Endoscopic cartilage tympanoplasty: Full thickness and partial thickness tragal graft. Braz. J. Otorhinolaryngol. 2019. [CrossRef]

11. Anschuetz, L.; Bonali, M.; Guarino, P.; Fabbri, F.B.; Alicandri-Ciufelli, M.; Villari, D.; Caversaccio, M.; Presutti, L. Management of Bleeding in Exclusive Endoscopic Ear Surgery: Pilot Clinical Experience. Otolaryngol. Head Neck Surg. 2017, 157, 700-706. [CrossRef]

12. Das, A.; Mitra, S.; Ghosh, D.; Kumar, S.; Sengupta, A. Does tranexamic acid improve intra-operative visualisation in endoscopic ear surgery? A double-blind, randomised, controlled trial. J. Laryngol. Otol. 2019. [CrossRef] [PubMed]

13. Swarup, A.; le Nobel, G.J.; Andrysek, J.; James, A.L. The Current Limitations and Future Direction of Instrument Design for Totally Endoscopic Ear Surgery: A Needs Analysis Survey. Otol. Neurotol. 2018, 39, 778-784. [CrossRef]

14. Schuknecht, H.F.; Gulya, A.J. Anatomy of the Temporal Bone with Surgical Implications; Lea \& Febiger: Philadelphia, PA, USA, 1986.

15. Badr-El-Dine, M.; James, A.L.; Panetti, G.; Marchioni, D.; Presutti, L.; Nogueira, J.F. Instrumentation and technologies in endoscopic ear surgery. Otolaryngol. Clin. N. Am. 2013, 46, 211-225. [CrossRef]

16. Kozin, E.D.; Kiringoda, R.; Lee, D.J. Incorporating Endoscopic Ear Surgery into Your Clinical Practice. Otolaryngol. Clin. N. Am. 2016, 49, 1237-1251. [CrossRef] [PubMed]

17. Mijovic, T.; Lea, J. Training and education in endoscopic ear surgery. Curr. Otorhinolaryngol. Rep. 2015, 3, 193-199. [CrossRef]

18. Alicandri-Ciufelli, M.; Molinari, G.; Beckmann, S.; Caversaccio, M.; Presutti, L.; Anschuetz, L. Epinephrine Use in Endoscopic Ear Surgery: Quantitative Safety Assessment. ORL 2019. [CrossRef]

19. Zhang, Y.; Bai, Y.; Chen, M.; Zhou, Y.; Yu, X.; Zhou, H.; Chen, G. The safety and efficiency of intravenous administration of tranexamic acid in coronary artery bypass grafting (CABG): A meta-analysis of 28 randomized controlled trials. BMC Anesthesiol. 2019, 19, 104. [CrossRef] [PubMed]

20. Cao, G.; Chen, G.; Huang, Q.; Huang, Z.; Alexander, P.G.; Lin, H.; Xu, H.; Zhou, Z.; Pei, F. The efficacy and safety of tranexamic acid for reducing blood loss following simultaneous bilateral total knee arthroplasty: A multicenter retrospective study. BMC Musculoskelet. Disord. 2019, 20, 325. [CrossRef]

21. Ping, W.D.; Zhao, Q.M.; Sun, H.F.; Lu, H.S.; Li, F. Role of tranexamic acid in nasal surgery: A systemic review and meta-analysis of randomized control trial. Medicine 2019, 98, e15202. [CrossRef]

22. Gilroy, A.M.; MacPherson, B.R.; Ross, L.M.; Broman, J.; Josephson, A. Atlas of Anatomy; Thieme Stuttgart: Stuttgart, Germany, 2008. 
23. Tomazic, P.V.; Hammer, G.P.; Gerstenberger, C.; Koele, W.; Stammberger, H. Heat development at nasal endoscopes' tips: Danger of tissue damage? A laboratory study. Laryngoscope 2012, 122, 1670-1673. [CrossRef]

24. Nelson, J.J.; Goyal, P. Temperature variations of nasal endoscopes. Laryngoscope 2011, 121, 273-278. [CrossRef] [PubMed]

25. MacKeith, S.A.; Frampton, S.; Pothier, D.D. Thermal properties of operative endoscopes used in otorhinolaryngology. J. Laryngol. Otol. 2008, 122, 711-714. [CrossRef] [PubMed]

26. Kozin, E.D.; Lehmann, A.; Carter, M.; Hight, E.; Cohen, M.; Nakajima, H.H.; Lee, D.J. Thermal effects of endoscopy in a human temporal bone model: Implications for endoscopic ear surgery. Laryngoscope 2014, 124, E332-E339. [CrossRef] [PubMed]

27. Aksoy, F.; Dogan, R.; Ozturan, O.; Eren, S.B.; Veyseller, B.; Gedik, O. Thermal effects of cold light sources used in otologic surgery. Eur. Arch. Otorhinolaryngol. 2015, 272, 2679-2687. [CrossRef]

28. Bottrill, I.; Perrault, D.F., Jr.; Poe, D. In vitro and in vivo determination of the thermal effect of middle ear endoscopy. Laryngoscope 1996, 106, 213-216. [CrossRef]

29. Craig, J.; Goyal, P. Insulating and cooling effects of nasal endoscope sheaths and irrigation. Int. Forum Allergy Rhinol. 2014, 4, 759-762. [CrossRef]

(C) 2020 by the authors. Licensee MDPI, Basel, Switzerland. This article is an open access article distributed under the terms and conditions of the Creative Commons Attribution (CC BY) license (http://creativecommons.org/licenses/by/4.0/). 